\title{
Dynamic interplay between viral adaptation and immune recognition during HIV-1 infection
}

\author{
Chihiro Motozono, Philip Mwimanzi, Takamasa Ueno ${ }^{\bowtie}$ \\ Center for AIDS Research, Kumamoto University, Kumamoto 860-0811, Japan \\ $\bowtie$ Correspondence: uenotaka@kumamoto-u.ac.jp \\ Received May 20, 2010 Accepted May 28, 2010
}

\begin{abstract}
Untreated human immunodeficiency virus (HIV) infections usually lead to death from AIDS, although the rate of the disease progression varies widely among individuals. The cytotoxic T lymphocyte (CTL) response, which is restricted by highly polymorphic MHC class I alleles, plays a central role in controlling HIV replication. It is now recognized that the antiviral efficacy of CTLs at the single cell level is dependent on their antigen specificity and is important in determining the quality of host response to viruses so that the individual will remain asymptomatic. However, because of the extreme mutational plasticity of HIV, HIV-specific CTL responses are continuously and dynamically changing. In order to rationally design an effective vaccine, the questions as to what constitutes an effective antiviral CTL response and what characterizes a potent antigenic peptide to induce such responses are becoming highlighted as needing to be answered.
\end{abstract}

KEYWORDS HIVIAIDS, peptide-MHC complex, HLA class I, cytotoxic T lymphocyte, immune escape

\section{INTRODUCTION}

Human immunodeficiency viruses (HIV) are the etiologic agents for acquired immunodeficiency syndrome (AIDS) in humans. HIV has spread to most parts of the world and constitutes the leading cause of death in many developing countries. HIV causes lifelong persistent infection in the host. An untreated HIV infection usually results in death from AIDS in about 10 years, although the rate of the disease progression varies widely among individuals. Especially, a small population of infected hosts (less than 1\%) appear to control HIV replication sufficiently well to remain asymptomatic. Such 'HIV controllers' or 'Elite controllers' have been under investigation in an attempt to identify what constitutes such an effective immune response to the virus (Baker et al., 2009; O'Connell et al., 2009).

Since the mid-1990s, antiretroviral therapy has improved and dramatically reduced HIV-related morbidity and mortality among those with access to treatment. However, only a small proportion of HIV-infected people in resource-limited countries can have access to such an effective antiretroviral therapy. Therefore, an effective HIV vaccine is urgently demanded. However, our understanding of $\mathrm{T}$ cell-mediated antiviral efficacy remains limited. Without such knowledge, vaccine design strategies will still remain empirical.

In this review, we will focus on the recent advances in our understanding of the efficacy and some limitations of the antiviral human immune system as well as that of the dynamic and shifting nature of the interplay between viral adaptation and host-specific selective pressure. Also, we discuss how the biochemical property of antigenic peptides influences the effectiveness of the host immune system.

\section{ROLES OF CD8+ T LYMPHOCYTES IN THE CONTROL OF HIV REPLICATION}

The human $\mathrm{MHC}$ region, located on chromosome 6 , is critical to the immune system, as it encodes proteins that play a central role in discrimination between self and non-self protein components. There are $3 \mathrm{MHC}$ class I loci in humans, i.e., HLA-A, HLA-B, and HLA-C, which are the most polymorphic loci in the entire human genome (Mungall et al., 2003). Almost all the encoded polymorphic residues are located in the peptide binding groove of the HLA class I (HLA-I) molecules, thereby defining the antigenic peptide repertoire that bind to these molecules (Rammensee et al., 1995). In relation to an HIV infection, a strong epidemiological link exists between specific HLA class I alleles and different rates of HIV disease progression (O'Brien et al., 2001). Also, more rapid disease 
progression is observed in individuals with HLA class I homozygosity (Carrington et al., 1999). These results suggest the significance of the antiviral immune system restricted by HLA-I alleles.

The virus-specific CD8+ cytotoxic T lymphocyte (CTL) is an important component of a host immune response toward HIV and plays a critical role in the containment of HIV replication during a course of an HIV infection (Borrow et al., 1994; Koup et al., 1994; Ogg et al., 1998). CTLs recognize HIV-infected cells by interaction of their own T cell receptors (TCRs) with viral peptides bound to HLA class I molecules on the cell surface of the infected cells and eliminate them directly by cytolysis or indirectly through the production of soluble factors such as interferon (IFN) $\gamma$ and various chemokines. Among these activities, the cytotoxic activity of CTLs toward HIV-infected cells is associated with efficient viral containment in vitro and in vivo (Yang et al., 1997; Migueles et al., 2002; SáezCirión n et al., 2007). However, HIV-specific CD8 T cell responses measured by the ability of these cells to bind with peptide-HLA class I tetrameric complexes (HLA tetramers) or to secrete IFN- $\gamma$ antigen specifically are correlated with the control of viremia in the acute phase (Borrow et al., 1994; Koup et al, 1994), but are not correlated in the case of chronic HIV-1 infections (Appay et al., 2000; Betts et al., 2001), indicating that a progressive functional defect occurs in HIV-specific CTLs during a chronic infection and that the efficacy or effectiveness of antiviral CTLs depends on qualitative rather than quantitative parameters. CTL quality and the control of human viral infections has been recently reviewed (Bangham, 2009).

\section{EFFECTS OF HIV GENETIC DIVERSITY ON ESCAPE FROM CTL RESPONSES}

One of the major characteristics of HIV is its extensive genetic variability. Within an individual, a progressive expansion of viral diversity occurs over the disease course (Shankarappa et al., 1999), with multiple variants co-existing as quasispecies that are unique to each patient. Over the natural course of an infection, the host immune response acts as a major selective force driving HIV evolution in a continuous dynamic process (Goulder and Watkins, 2004). Despite the extreme mutational capacity of the virus, there are constraints on viral evolution; and escape in response to specific immune selective pressures follows broadly predictable mutation patterns (Allen et al., 2005a). Among the host immune responses, the CTL is a potentially major selective force in vivo driving genome-wide viral evolution (Carlson and Brumme, 2008). Genetic variation in the polymorphic HLA-I alleles contributes to diversity of pathogen recognition by CTLs and acts as a selective force shaping viral evolution in an HIV-infected individual through selection of mutations that allow the virus to escape from CTL recognition.

\section{CHANGES IN CTL SPECIFICITY OVER TIME}

It is becoming evident that HIV-specific CTL responses are shifted in HIV-infected individuals during the transition from the early to the chronic phase of an HIV-1 infection (Fig. 1A) (Goulder et al., 2001; Allen et al., 2005b; Feeney et al., 2005; Ueno et al., 2007, 2008). For example, in longitudinally looking at the HLA-B35-restricted CD8+ T cell responses in HIV-infected individuals, the Nef VY8 epitope (VPLRPMTY) is dominantly recognized by CTLs relatively early in an infection; whereas the N-terminal extended RY11 epitope (RPQVPLRPMTY) is recognized by CTLs in the chronic phase (Ueno et al., 2008). Also, an immunodominant response directed against the HIV Gag p17 derived, HLA-A0201 restricted SL9 epitope (SLYNTVATL) is detected in the chronic phase but rarely detected in the early phase of an infection (Goulder et al., 2001). Although the mechanisms underlying this phenomenon are not yet known, it is conceivable that the responses detected in the early stage of infection could have mutated away, opening the field for a second wave of CTL specificities taking their place. The CTLs induced by a second or third waves of CTL specificities possess less efficient antiviral activity. In the case of the HLA-B35-restricted CTL responses mentioned above, the highly active VY8-specific CTLs elicited early in an infection become ineffective due to the acquisition of the Tyr85 to Phe Nef mutation by the virus; and subsequently the cross-reactive RY11-specific CTLs, yet having moderate antiviral activity, become dominant (Ueno et al., 2008). Furthermore, the Arg75 to Thr variant, which has been selected by RY11-specific CTLs during the chronic phase, can induce the variant-specific CTLs de novo with less effective antigen-specific proliferative capacity, further reducing the antiviral activity of CTLs in vivo (Ueno et al., 2007).

As such, the generation of antiviral CTL responses and the selection of CTL escape variants are intertwined with each other at any given time during an HIV infection. However, because the newly-generated $T$ cell responses somewhat exhibit an altered functional profile, which leads to less efficient antiviral activity, the gross antiviral CTL activity toward HIV in an individual decreases over time during the course of the infection.

\section{IMMUNOGENICITY OF CTL EPITOPES}

As mentioned above, the antiviral activity of CTLs is not equally potent but is dependent on their antigen specificity (Fig. 1B) (Yang et al., 2003; Ueno et al., 2004; Tomiyama et al., 2005; Ueno et al., 2007). Then the fundamental question can be raised as to what factors govern the generation of effective antiviral CTL responses to a viral infection and how they do so. The capability of an antigenic peptide to evoke an effective CTL response is termed immunogenicity or antigenicity. The factors that influence immunogenicity of antigenic peptides have been extensively analyzed mostly 
A

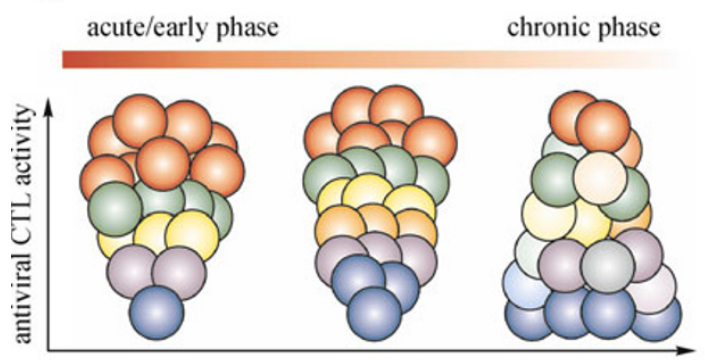

time after infection
B

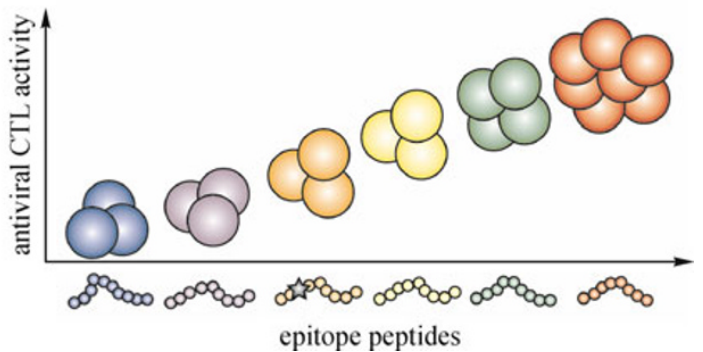

Figure 1. Dynamic changes in CTL specificity and functional hierarchy over time. (A) Antiviral CTL responses observed in the acute/early phase of an HIV infection are dominantly composed of a fraction of CTLs with potent antiviral activity; whereas those observed in the chronic phase are mostly comprise another fraction of CTLs with moderate or weak antiviral activity. Circles represent HIV-specific CTLs, and colors indicate the differences in antigenic peptides recognized by these CTLs. (B) Antiviral activity of CTLs is different dependent on their antigen specificity, including the antigen with a CTL escape mutation (shown as a star).

based on the mouse model (Yewdell, 2006). The immunogenicity is influenced by several factors including kinetics and amplitude of antigenic protein expression, antigen processing and processing machinery, the preferences of the peptide for binding to a given HLA-I molecule, limitations in the diversity of the TCR repertoire, etc (see review by Yewdell (2006) for details). In addition, because the binding kinetics between TCR and pMHC greatly influences $\mathrm{T}$ cell activation, the interplay between the structural and immunological characterization of antigens has particular clinical interests with respect to the design of peptide-based vaccines (Apostolopoulos et al., 2008). For example, the structure of HLA-B57 in complex with several HIV Gag peptides, which are known to be associated with long-term nonprogression during an HIV infection, has been solved (Stewart-Jones et al., 2005). Such studies are particularly important to provide detailed information on the nature of the antigenic peptides accommodated in the peptide binding groove of HLA-I molecules; however, it is not yet possible to predict the immunogenic potency of a given antigenic peptide from its crystal structure or amino acid sequence (Rudolph et al., 2006; Yewdell, 2006).

Both the immunogenicity of antigenic peptides and the antiviral activity of CTLs stem from multifactorial events, reflecting a consequence of various positive and negative factors that govern viral replication, antigen presentation and $\mathrm{T}$ cell activation. Broad comparisons between very different virus strains, peptide antigens, MHCs, and TCRs provide little information beyond highlighting just the differences. Comparisons between more closely related viral antigens and $\mathrm{MHCs}$ could be more revealing. In this regard, Motozono et al. (2009) performed detailed analysis of 2 closely related HIV Nef-derived antigens, VY8 (VPLRPMTY) and RY11 (RPQVPLRPMTY), presented by the same HLA-B35 molecule. The effective antiviral CTL activity was influenced by the peptide-off rate from the pMHC complex on the target cell surface (Fig. 2). The introduction of a mutation in the middle of the peptides delayed $\mathrm{pMHC}$ decay and rendered the target cells more susceptible to CTL killing (Motozono et al., 2009), suggesting that the strategy of altered peptide ligand is capable of enhancing CTL-mediated immune responses against HIV-1 infection, similar to that used for anti-cancer vaccines targeting self antigens (Yu et al., 2004; Borbulevych et al., 2005). Moreover, analysis of the pMHC heterotrimer complexes by differential scanning calorimetry and circular dichroism show very different thermostability profiles dependent on the antigenic peptides and reveal that interdependent and cooperative thermodynamic profiles of $\mathrm{pMHC}$ can be associated with efficient recognition by CTLs for killing virusinfected target cells (Motozono et al., 2009). The importance of thermal unfolding pattern has been reported for various self peptides in complex with HLA-B27, some of which are associated with autoimmune diseases (Hillig et al., 2004; Hülsmeyer et al., 2005). These results highlight the importance of incorporating thermostability data in the process of rational optimization of antigens that support profound antiviral activity by HIV-specific CTLs (Fig. 2).

\section{SUMMARY AND PERSPECTIVES}

Overall, multiple lines of investigation are necessary to fully understand what constitutes effective antiviral CTL responses because of the highly variable nature of the human immune system and of viral genes. However, the availability of large cohorts of HIV-infected individuals holds a potential goldmine for comprehensive analysis and monitoring of immunogenicity of antigenic peptides and effectiveness of their cognate CTLs in humans. Because of the high variation of HIV proteins, not only antiviral cytotoxic activity of CTLs but also 


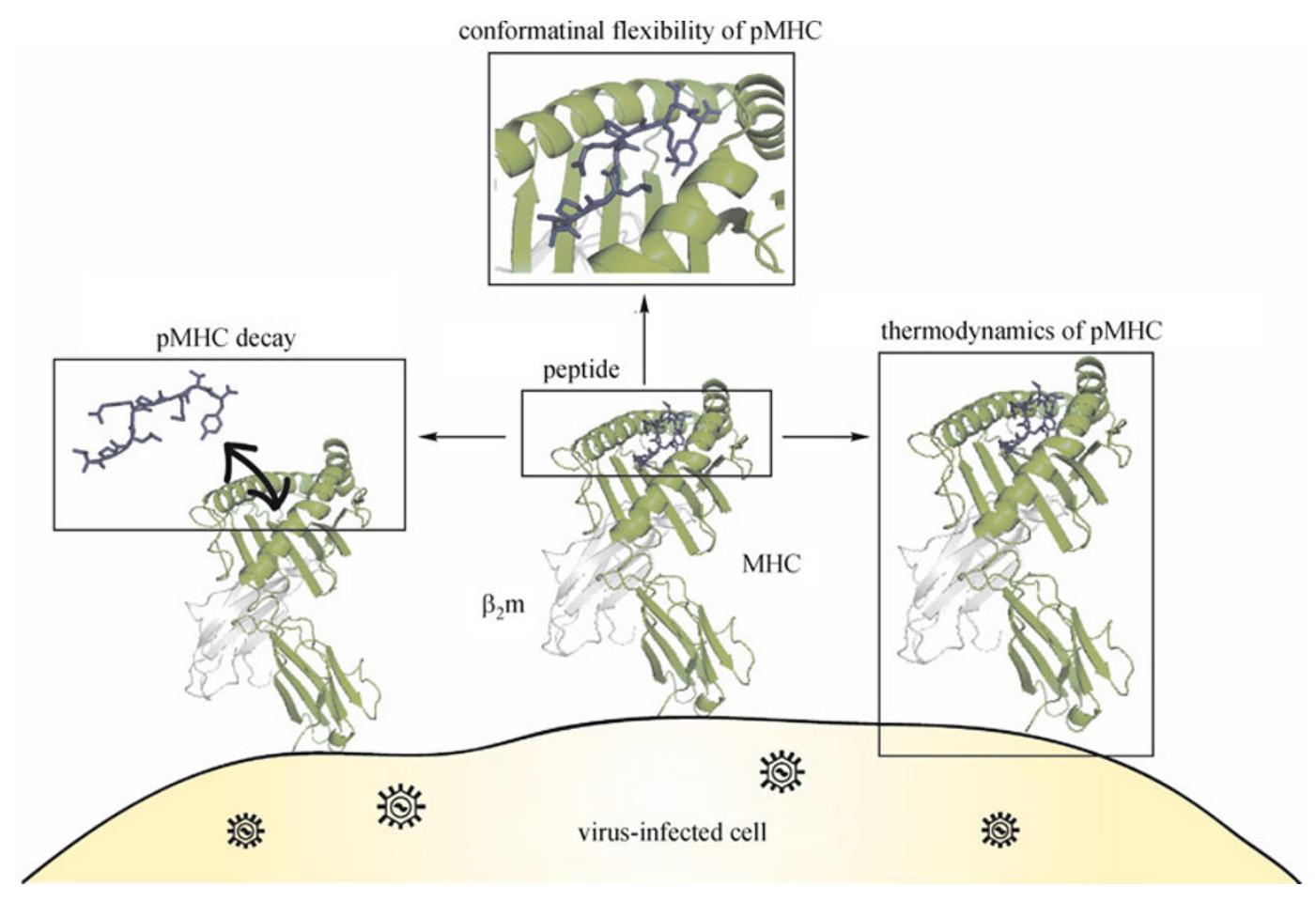

Figure 2. Possible peptide intrinsic factors affecting CTL antiviral activity. Viral peptide, $\beta_{2}$ microglobulin $\left(\beta_{2} \mathrm{~m}\right)$, and MHC heavy chain are colored blue, gray, and green, respectively. The configuration of the pMHC structure is reconstructed according to the coordinates of HLA-B*3501 in complex with the HIV Nef VY8 peptide (Smith et al., 1996).

cross-reactive capacity of CTLs toward various variant viruses may influence the efficacy of CTL responses for the control of viral replication. In this regard, by combining cohort study and bioinformatics approaches, Kosmrlj et al. (2010) recently reported that individuals with a more cross-reactive CTL repertoire control viral replication better during the acute phase of the infection and that the thymic selection step mediated by the HLA-I molecules in complex with self peptides may influence the cross-reactive potency of the mature T cell repertoire. Moreover, Borbulevych et al. (2009) showed that antigen-dependent tuning of molecular motion can contribute to $T$ cell recognition and facilitate $T$ cell crossreactivity. The peptide in association with a certain HLA-I molecule thus plays an important role in mediating effective antiviral activity and cross-reactive capacity of CTLs (Fig. 2). Further biochemical approaches to shed new light on the intrinsic characteristics of pMHC are increasingly becoming warranted.

\section{ACKNOWLEDGEMENTS}

This research was supported in part by a grant-in-aid for scientific research from the Ministry of Education, Science, Sports, and Culture of Japan, by a grant from Human Science Foundation, and by a grantin-aid for AIDS research from the Ministry of Health, Labor, and Welfare of Japan.

\section{REFERENCES}

Allen, T.M., Altfeld, M., Geer, S.C., Kalife, E.T., Moore, C., O'sullivan, K.M., Desouza, I., Feeney, M.E., Eldridge, R.L., Maier, E.L., et al. (2005a). Selective escape from CD8+ T-cell responses represents a major driving force of human immunodeficiency virus type 1 (HIV1) sequence diversity and reveals constraints on HIV-1 evolution. J Virol 79, 13239-13249.

Allen, T.M., Yu, X.G., Kalife, E.T., Reyor, L.L., Lichterfeld, M., John, M., Cheng, M., Allgaier, R.L., Mui, S., Frahm, N., et al. (2005b). De novo generation of escape variant-specific CD8+ T-cell responses following cytotoxic T-lymphocyte escape in chronic human immunodeficiency virus type 1 infection. J Virol 79, 12952-12960.

Apostolopoulos, V., Lazoura, E., and Yu, M. (2008). MHC and MHClike molecules: structural perspectives on the design of molecular vaccines. Adv Exp Med Biol 640, 252-267.

Appay, V., Nixon, D.F., Donahoe, S.M., Gillespie, G.M., Dong, T., King, A., Ogg, G.S., Spiegel, H.M., Conlon, C., Spina, C.A., et al. (2000). HIV-specific CD8(+) T cells produce antiviral cytokines but are impaired in cytolytic function. J Exp Med 192, 63-75.

Baker, B.M., Block, B.L., Rothchild, A.C., and Walker, B.D. (2009). Elite control of HIV infection: implications for vaccine design. Expert Opin Biol Ther 9, 55-69.

Bangham, C.R.M. (2009). CTL quality and the control of human retroviral infections. Eur J Immunol 39, 1700-1712.

Betts, M.R., Ambrozak, D.R., Douek, D.C., Bonhoeffer, S., Brenchley, J.M., Casazza, J.P., Koup, R.A., and Picker, L.J. (2001). Analysis 
of total human immunodeficiency virus (HIV)-specific CD4(+) and CD8(+) T-cell responses: relationship to viral load in untreated HIV infection. J Virol 75, 11983-11991.

Borbulevych, O.Y., Baxter, T.K., Yu, Z., Restifo, N.P., and Baker, B.M. (2005). Increased immunogenicity of an anchor-modified tumorassociated antigen is due to the enhanced stability of the peptide/ MHC complex: implications for vaccine design. J Immunol 174, 4812-4820.

Borbulevych, O.Y., Piepenbrink, K.H., Gloor, B.E., Scott, D.R., Sommese, R.F., Cole, D.K., Sewell, A.K., and Baker, B.M. (2009). T cell receptor cross-reactivity directed by antigendependent tuning of peptide-MHC molecular flexibility. Immunity 31, 885-896.

Borrow, P., Lewicki, H., Hahn, B.H., Shaw, G.M., and Oldstone, M.B. (1994). Virus-specific CD8+ cytotoxic T-lymphocyte activity associated with control of viremia in primary human immunodeficiency virus type 1 infection. J Virol 68, 6103-6110.

Carlson, J.M., and Brumme, Z.L. (2008). HIV evolution in response to HLA-restricted CTL selection pressures: a population-based perspective. Microbes Infect 10, 455-461.

Carrington, M., Nelson, G.W., Martin, M.P., Kissner, T., Vlahov, D., Goedert, J.J., Kaslow, R., Buchbinder, S., Hoots, K., and O'Brien, S.J. (1999). HLA and HIV-1: heterozygote advantage and $B^{\star} 35-$ $\mathrm{Cw}^{*} 04$ disadvantage. Science 283, 1748-1752.

Feeney, M.E., Tang, Y., Pfafferott, K., Roosevelt, K.A., Draenert, R., Trocha, A., Yu, X.G., Verrill, C., Allen, T., Moore, C., et al. (2005). HIV-1 viral escape in infancy followed by emergence of a variantspecific CTL response. J Immunol 174, 7524-7530.

Goulder, P.J.R., Altfeld, M.A., Rosenberg, E.S., Nguyen, T., Tang, Y., Eldridge, R.L., Addo, M.M., He, S., Mukherjee, J.S., Phillips, M.N., et al. (2001). Substantial differences in specificity of HIV-specific cytotoxic T cells in acute and chronic HIV infection. J Exp Med 193, 181-194.

Goulder, P.J.R., and Watkins, D.I. (2004). HIV and SIV CTL escape: implications for vaccine design. Nat Rev Immunol 4, 630-640.

Hillig, R.C., Hülsmeyer, M., Saenger, W., Welfle, K., Misselwitz, R., Welfle, H., Kozerski, C., Volz, A., Uchanska-Ziegler, B., and Ziegler, A. (2004). Thermodynamic and structural analysis of peptide- and allele-dependent properties of two HLA-B27 subtypes exhibiting differential disease association. J Biol Chem 279, 652-663.

Hülsmeyer, M., Welfle, K., Pöhlmann, T., Misselwitz, R., Alexiev, U., Welfle, H., Saenger, W., Uchanska-Ziegler, B., and Ziegler, A. (2005). Thermodynamic and structural equivalence of two HLAB27 subtypes complexed with a self-peptide. J Mol Biol 346, 1367-1379.

Kosmrlj, A., Read, E.L., Qi, Y., Allen, T.M., Altfeld, M., Deeks, S.G., Pereyra, F., Carrington, M., Walker, B.D., and Chakraborty, A.K. (2010). Effects of thymic selection of the T-cell repertoire on HLA class I-associated control of HIV infection. Nature 465, 350-354.

Koup, R.A., Safrit, J.T., Cao, Y., Andrews, C.A., McLeod, G., Borkowsky, W., Farthing, C., andHo, D.D. (1994). Temporal association of cellular immune responses with the initial control of viremia in primary human immunodeficiency virus type 1 syndrome. J Virol 68, 4650-4655.

Migueles, S.A., Laborico, A.C., Shupert, W.L., Sabbaghian, M.S., Rabin, R., Hallahan, C.W., Van Baarle, D., Kostense, S., Miedema, F., McLaughlin, M., et al. (2002). HIV-specific CD8+ T cell proliferation is coupled to perforin expression and is maintained in nonprogressors. Nat Immunol 3, 1061-1068.

Motozono, C., Yanaka, S., Tsumoto, K., Takiguchi, M., and Ueno, T. (2009). Impact of intrinsic cooperative thermodynamics of peptideMHC complexes on antiviral activity of HIV-specific CTL. J Immunol 182, 5528-5536.

Mungall, A.J., Palmer, S.A., Sims, S.K., Edwards, C.A., Ashurst, J.L., Wilming, L., Jones, M.C., Horton, R., Hunt, S.E., Scott, C.E., et al. (2003). The DNA sequence and analysis of human chromosome 6. Nature 425, 805-811.

O'Brien, S.J., Gao, X., and Carrington, M. (2001). HLA and AIDS: a cautionary tale. Trends Mol Med 7, 379-381.

O'Connell, K.A., Bailey, J.R., and Blankson, J.N. (2009). Elucidating the elite: mechanisms of control in HIV-1 infection. Trends Pharmacol Sci 30, 631-637.

Ogg, G.S., Jin, X., Bonhoeffer, S., Dunbar, P.R., Nowak, M.A., Monard, S., Segal, J.P., Cao, Y., Rowland-Jones, S.L., Cerundolo, V., et al. (1998). Quantitation of HIV-1-specific cytotoxic T lymphocytes and plasma load of viral RNA. Science 279, 2103-2106.

Rammensee, H.G., Friede, T., and Stevanoviíc, S. (1995). MHC ligands and peptide motifs: first listing. Immunogenetics 41, 178-228.

Rudolph, M.G., Stanfield, R.L., and Wilson, I.A. (2006). How TCRs bind MHCs, peptides, and coreceptors. Annu Rev Immunol 24, 419-466.

Sáez-Cirión, A., Lacabaratz, C., Lambotte, O., Versmisse, P., Urrutia, A., Boufassa, F., Barré-Sinoussi, F., Delfraissy, J.-F., Sinet, M., Pancino, G., et al., and the Agence Nationale de Recherches sur le Sida EP36 HIV Controllers Study Group. (2007). HIV controllers exhibit potent CD8 T cell capacity to suppress HIV infection ex vivo and peculiar cytotoxic $T$ lymphocyte activation phenotype. Proc Natl Acad Sci U S A 104, 6776-6781.

Shankarappa, R., Margolick, J.B., Gange, S.J., Rodrigo, A.G., Upchurch, D., Farzadegan, H., Gupta, P., Rinaldo, C.R., Learn, G.H., He, X., et al. (1999). Consistent viral evolutionary changes associated with the progression of human immunodeficiency virus type 1 infection. J Virol 73, 10489-10502.

Smith, K.J., Reid, S.W., Stuart, D.I., McMichael, A.J., Jones, E.Y., and Bell, J.I. (1996). An altered position of the $\alpha 2$ helix of MHC class I is revealed by the crystal structure of HLA-B*3501. Immunity 4 , 203-213.

Stewart-Jones, G.B.E., Gillespie, G., Overton, I.M., Kaul, R., Roche, P., McMichael, A.J., Rowland-Jones, S., and Jones, E.Y. (2005). Structures of three HIV-1 HLA-B*5703-peptide complexes and identification of related HLAs potentially associated with long-term nonprogression. J Immunol 175, 2459-2468.

Tomiyama, H., Fujiwara, M., Oka, S., and Takiguchi, M. (2005). Cutting Edge: Epitope-dependent effect of Nef-mediated HLA class I down-regulation on ability of HIV-1-specific CTLs to suppress HIV-1 replication. J Immunol 174, 36-40.

Ueno, T., Idegami, Y., Motozono, C., Oka, S., and Takiguchi, M. (2007). Altering effects of antigenic variations in HIV-1 on antiviral effectiveness of HIV-specific CTLs. J Immunol 178, 5513-5523.

Ueno, T., Motozono, C., Dohki, S., Mwimanzi, P., Rauch, S., Fackler, O.T., Oka, S., and Takiguchi, M. (2008). CTL-mediated selective pressure influences dynamic evolution and pathogenic functions of HIV-1 Nef. J Immunol 180, 1107-1116.

Ueno, T., Tomiyama, H., Fujiwara, M., Oka, S., and Takiguchi, M. 
(2004). Functionally impaired HIV-specific CD8 T cells show high affinity TCR-ligand interactions. J Immunol 173, 5451-5457.

Yang, O.O., Kalams, S.A., Trocha, A., Cao, H., Luster, A., Johnson, R.P., and Walker, B.D. (1997). Suppression of human immunodeficiency virus type 1 replication by CD8+ cells: evidence for HLA class I-restricted triggering of cytolytic and noncytolytic mechanisms. J Virol 71, 3120-3128.

Yang, O.O., Sarkis, P.T.N., Trocha, A., Kalams, S.A., Johnson, R.P., and Walker, B.D. (2003). Impacts of avidity and specificity on the antiviral efficiency of HIV-1-specific CTL. J Immunol 171, 3718-3724.

Yewdell, J.W. (2006). Confronting complexity: real-world immunodominance in antiviral CD8+ T cell responses. Immunity 25, 533-543.

Yu, Z., Theoret, M.R., Touloukian, C.E., Surman, D.R., Garman, S.C., Feigenbaum, L., Baxter, T.K., Baker, B.M., and Restifo, N.P. (2004). Poor immunogenicity of a self/tumor antigen derives from peptide-MHC-I instability and is independent of tolerance. J Clin Invest 114, 551-559. 